\title{
Antarctic site testing - microthermal measurements of surface-layer seeing at the South Pole
}

\author{
R.D. Marks ${ }^{1}$, J. Vernin ${ }^{2}$, M. Azouit ${ }^{2}$, J.W. Briggs ${ }^{3}$, M.G. Burton ${ }^{1}$, M.C.B. Ashley ${ }^{1}$ and J.F. Manigault ${ }^{2}$ \\ 1 Joint Australian Centre for Astrophysical Research in Antarctica, School of Physics, University of New South Wales, \\ Sydney 2052, Australia \\ 2 Département d'Astrophysique de l'Université de Nice, U.R.A. 709 du C.N.R.S., F-06108 Nice Cedex 2, France \\ 3 Center for Astrophysical Research in Antarctica, University of Chicago, Yerkes Observatory, Williams Bay, WI \\ 53191, U.S.A.
}

Received October 23, 1995; accepted January 15, 1996

\begin{abstract}
Results from experiments measuring the optical seeing in the surface layer at the South Pole Station are presented. Seeing measurements were taken over 49 data runs between April and August 1994, using microthermal sensors placed at 3 levels on a $27 \mathrm{~m}$-high mast. The seeing contribution from this region was quite large in comparison with similar experiments performed at other sites, with a mean value measured over this period of $0.64^{\prime \prime}$. However, there is often a significant decrease in the optical turbulence over the height of the mast, with mean values of $0.37^{\prime \prime}$ and $0.46^{\prime \prime}$ measured in the upper $(17-27 \mathrm{~m})$ and lower $(7-17 \mathrm{~m})$ sections respectively. These measurements coincide with a large and highly variable temperature inversion, the behaviour of which is often well correlated with the observed turbulence profile. The results can be roughly separated into four or five categories, characterised by the temperature inversion alone. Further analysis of the data should provide some predictive power about the likely optical turbulence profile of the surface layer in given observing conditions.
\end{abstract}

Key words: atmospheric effects — instrumentation: miscellaneous — methods: observational — site testing

\section{Introduction}

There has been increased recognition, in recent years, of the potential quality of the antarctic plateau as an astronomical site. The dry, cold air, high altitude, low surface wind speeds, low integrated water vapour, as well as the ability to perform long continuous observations, all point to the possibility that an observatory stationed on the high inland regions of the continent could out-perform observatories at any other site on earth (Vernin 1994; Burton et al. 1994; Ashley et al. 1995b).

This has led to the development of a site-testing program, a collaboration between the University of New South Wales (Australia), the Université de Nice (France) and the Center for Astrophysical Research in Antarctica (U.S.A.), at the Amundsen-Scott South Pole Station. This program consists of a suite of experiments measuring the seeing contribution of the surface layer and free atmosphere, and other instruments (both planned and currently operational, see, e.g., Vernin et al. 1994; Ashley et al. 1995a) measuring the atmospheric transmission from the IR to the near-UV regions of the spectrum.

Send offprint requests to: $\mathrm{R}$. Marks
The South Pole, due to its location "downhill" from the highest points on the plateau, may not be the best site in Antarctica. It is expected, however, to give an indication of the properties of the atmosphere on the plateau in general, before similar site testing experiments can be performed at more favourable, but currently inaccessible, sites, such as Domes A and C (see Storey et al. 1995). The aim of the project reported here is to measure the image degradation caused by atmospheric turbulence above the South Pole. It is expected that the free atmosphere may reveal exceptional seeing (Gillingham 1993; Vernin 1994). The reasons for this include the negligible diurnal variation, low surface wind speeds in the higher regions of the plateau, the circumpolar circulation patterns (resulting in the absence of strong wind shears in the upper atmosphere), as well as the low tropopause over polar regions ( $~ 8 \mathrm{~km}$, Schwerdtfeger 1984). However, it is known that very large and unstable temperature inversions may occur in the surface layer, up to an altitude of $\sim 100 \mathrm{~m}$, so that this layer will be of more significance to the seeing than at other sites.

The experiment has been divided into two parts: measurement of 
1. the seeing contribution of the atmospheric surface layer during the 1994 season, and

2. the contribution from the boundary layer and free atmosphere, being carried out through the 1995 season, using balloon-borne microthermal sensors.

This paper presents the results from the surface-layer experiments, which were performed using a $27 \mathrm{~m}$-high mast in place at the Station. 49 data runs were obtained over the course of the 1994 winter, between April and August. These data allow us to determine the overall seeing produced in this $27 \mathrm{~m}$ layer, as well as the vertical profile of the turbulence strength. Temperature, pressure and wind speed measurements taken from the same mast were used to check the calibration of our instruments, and also allow some comments on the seeing caused by this layer in different weather conditions.

\section{Experimental method}

\subsection{Principles of seeing measurement}

The theory of atmospheric turbulence (e.g., Tatarski 1961), and in particular its relation to astronomical seeing (Roddier 1981), is very well established. The "Fried parameter", $r_{0}$, represents the telescope aperture diameter, for which the diffraction-limited image resolution is equal to the FWHM of the seeing-limited image. The relationship between this parameter and atmospheric turbulence was determined by Fried (1966) to be:

$$
r_{0}=\left(16.7 \lambda^{-2} \int_{0}^{\infty} C_{N}^{2}(h) \mathrm{d} h\right)^{-3 / 5}
$$

where $h$ is the height through the atmosphere, $C_{N}^{2}(h)$ is the refractive index structure constant and $\lambda$ the wavelength.

Following the work of Roddier (1981), the angular size in arcseconds of the FWHM of the images point spread function is given by Dierickx (1992) as:

$$
\varepsilon_{\mathrm{fwhm}}=0.98 \frac{\lambda}{r_{0}}
$$

and hence:

$$
\varepsilon_{\mathrm{fwhm}}=5.25 \lambda^{-1 / 5}\left(\int_{0}^{\infty} C_{N}^{2}(h) \mathrm{d} h\right)^{3 / 5}
$$

The refractive index structure constant in this case can be taken to represent the sum of the contributions from all turbulent layers in the atmosphere. These can be directly related to the corresponding temperature structure constant as follows:

$$
C_{N}^{2}(h)=\left(80.10^{-6} \frac{P(h)}{T(h)^{2}}\right)^{2} C_{T}^{2}(h)
$$

where $\mathrm{P}(\mathrm{h})$ is the pressure and $\mathrm{T}(\mathrm{h})$ the temperature.
The method used to determine $C_{N}^{2}$ involves measurement of the temperature structure function, $D_{T}(r, h)$, defined as the mean square of the temperature fluctuations between two points separated by $r$, where $r$ lies between the inner and outer scale of the turbulent motion. As derived by Obukhov (1949), this is related to $C_{T}^{2}(h)$ by:

$$
D_{T}(r, h)=C_{T}^{2}(h) r^{2 / 3}
$$

\subsection{Instrument design}

The experiment was performed using the tallest mast available at the South Pole Station, a 27 m-high mast set up by the National Oceanographic Atmospheric Administration (NOAA) for meteorological measurements. This mast is located on the up-wind side of the Station (the wind direction being in general from within a quadrant facing towards the higher regions of the plateau), and hence the effect of heat generated by the base itself is minimal.

$C_{T}^{2}$ measurements were taken using pairs of microthermal sensors ${ }^{1}$, the resistance of which vary in proportion to the very small and rapid temperature fluctuations associated with the turbulence. The sensors were placed at three levels on the mast, at 7, 17 and $27 \mathrm{~m}$ above the surface. Additional sensors were placed at each level to measure the temperature differences and variation between levels, as well as the absolute temperature at the $17 \mathrm{~m}$ level. We also had access to the NOAA data from the mast during our data runs; in particular the temperature at 2 and $20 \mathrm{~m}$, wind speed and direction, and pressure. These data were used to perform the $C_{T}^{2}$ to $C_{N}^{2}$ computations (see Eq. 4), as well as providing an additional check on the accuracy of our vertical temperature profile.

The $C_{N}^{2}$ measurements tend to decrease sharply and nonlinearly with altitude, and so the calculation of the seeing produced between each pair of levels on the mast was performed (via Eq. 3) assuming a power law variation with altitude over each $10 \mathrm{~m}$ slice.

The sensor couples were placed on horizontal beams pointing in the upwind direction, to avoid the possibility of local effects due to the mast. In addition, two pairs of sensors were used at each level, separated from each other by $\sim 2 \mathrm{~m}$, to give an additional check on the reliability of the data in the case of unusual weather conditions.

The sensors were connected by cables back to the Clean Air Building (the centre for NOAA operations), some $100 \mathrm{~m}$ away. The signal processing equipment used was similar to experiments done to measure microthermal turbulence at other sites (Vernin \& Muñoz-Tunón 1992). The raw sensor voltage output is sent to an amplifier which also performs the mean-square calculation of $D_{T}^{2}$, then on

\footnotetext{
${ }^{1}$ The sensors and electronic instrumentation we deployed at the South Pole were developed by M. Azouit and J.F. Manigault at the Département d'Astrophysique, Université de Nice.
} 
to a $\mathrm{PC}$, using a 16-channel $\mathrm{A} / \mathrm{D}$ converter to digitise the data.

Data were recorded from all sensors at intervals of $1 \mathrm{~s}$, and integrated over $60 \mathrm{~s}$. Data runs were performed by one of us (JWB) at irregular intervals throughout the season, in a wide range of weather and cloud-cover conditions.

The durations of the data runs were limited due to ice formation on the microthermal sensors in some weather conditions. This problem was overcome by the use of tin covers to protect the sensors when not in use, and by a small current being passed through the filaments to sublimate the ice between data runs. The presence of ice could be readily determined by noting where the signal from the detectors decreased due to their increased time constant.

\section{Results}

Microthermal data from the mast were taken on a total of 49 occasions during the 1994 winter, between April 26 and August 10. The duration of the runs varied from 1 to 14 hrs depending on the condition of the sensors. Any data where ice on the sensors appeared to have had an effect were rejected. The data runs took place under a wide variety of weather conditions, in terms of wind, cloud cover, temperature and temperature inversion. For the purpose of calculating an average value for the seeing, only the first hour of integrated data was used from each run, so as to avoid a bias towards the weather conditions where long runs were possible. These results are shown in Table 1 .

Table 1. Turbulence contributions to surface layer seeing $(7-27 \mathrm{~m})$, for separate and combined levels, measured on the NOAA mast over 49 data runs between 26 April and 10 August 1994

\begin{tabular}{lll}
\hline Height range & $\begin{array}{l}\bar{\varepsilon} \\
(\operatorname{arcsecs})\end{array}$ & $\begin{array}{l}\text { Median } \\
(\operatorname{arcsecs})\end{array}$ \\
\hline lower $(7-17 \mathrm{~m})$ & 0.46 & 0.42 \\
upper $(17-27 \mathrm{~m})$ & 0.37 & 0.31 \\
total $(7-27 \mathrm{~m})$ & 0.64 & 0.59 \\
\hline
\end{tabular}

It should be noted that the seeing contributions from the two levels do not add linearly since from Eq. (3):

$$
\varepsilon_{\text {total }}=\left(\sum_{i} \varepsilon_{i}^{5 / 3}\right)^{3 / 5}
$$

The results are summarised in Fig. 1, which shows the probability distribution of seeing for the combined levels and for the two levels separately, and the associated cumulative distributions.

Temperature inversions measured with our data range from $0-9^{\circ} \mathrm{C}$ between 7 and $27 \mathrm{~m}$, with large variations in
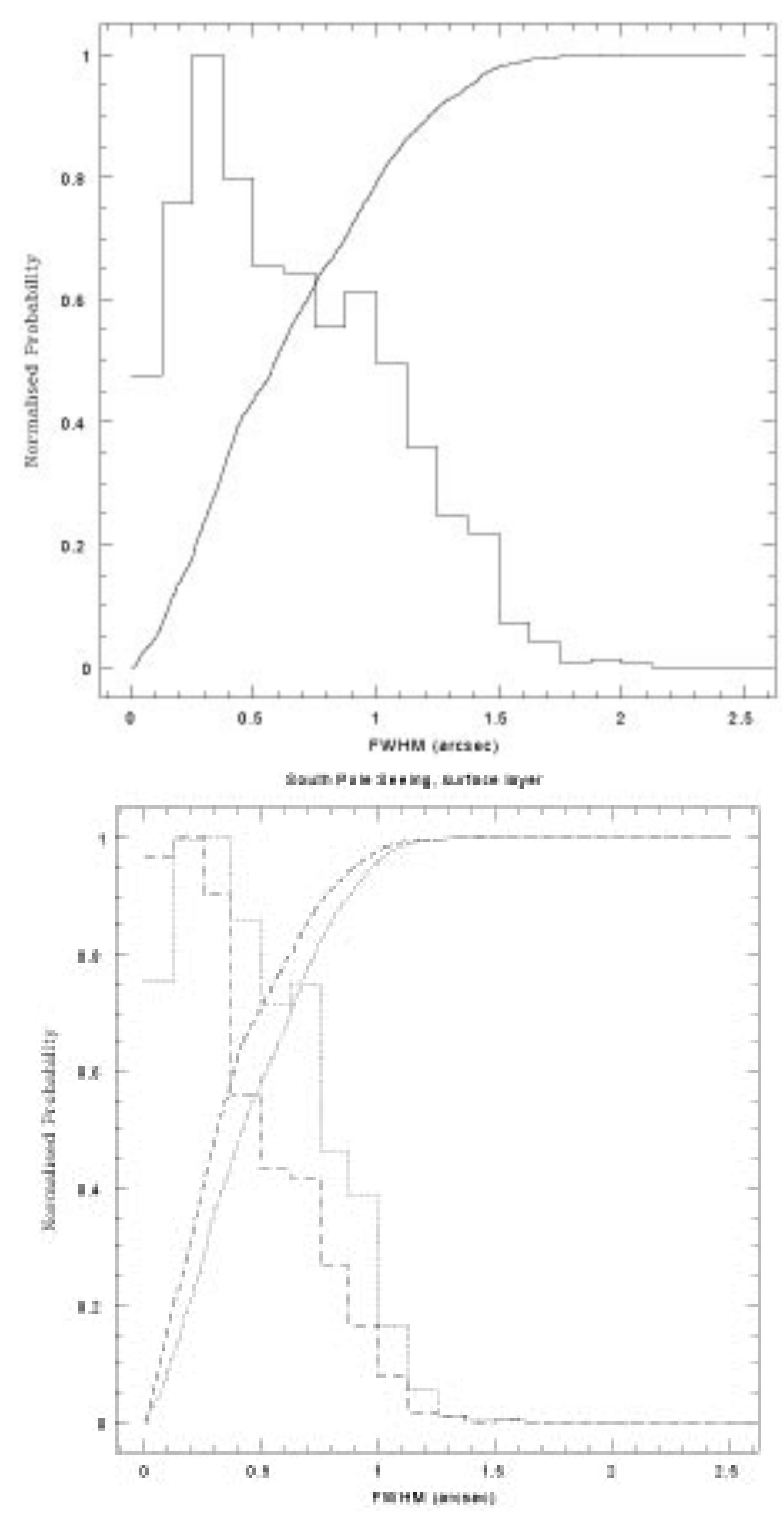

Fig. 1a and b. Seeing data statistics: FWHM probability distribution and associated cumulative distribution for: a) combined levels (i.e., $7-27 \mathrm{~m}$ ), b) the two separate levels, $7-17 \mathrm{~m}$ (dotted line) and 17-27 $\mathrm{m}$ (dashed line)

the relative contribution from each of the two levels. No negative temperature gradients were observed.

The correlation between the readings of the two pairs of sensors at each level is very good, with a correlation function of $>0.9$ generally being obtained. Due to the widely separated positions and relative orientations of the sensors, this indicates that local effects from the mast or the sensor supports themselves are virtually absent, and the readings from each sensor couple are a true measurement of the undisturbed atmospheric turbulence.

These results show that the contribution of the surface layer to the overall seeing is quite significant, in contrast to sites such as La Palma (Vernin \& Tuñon-Muñóz 1994) 

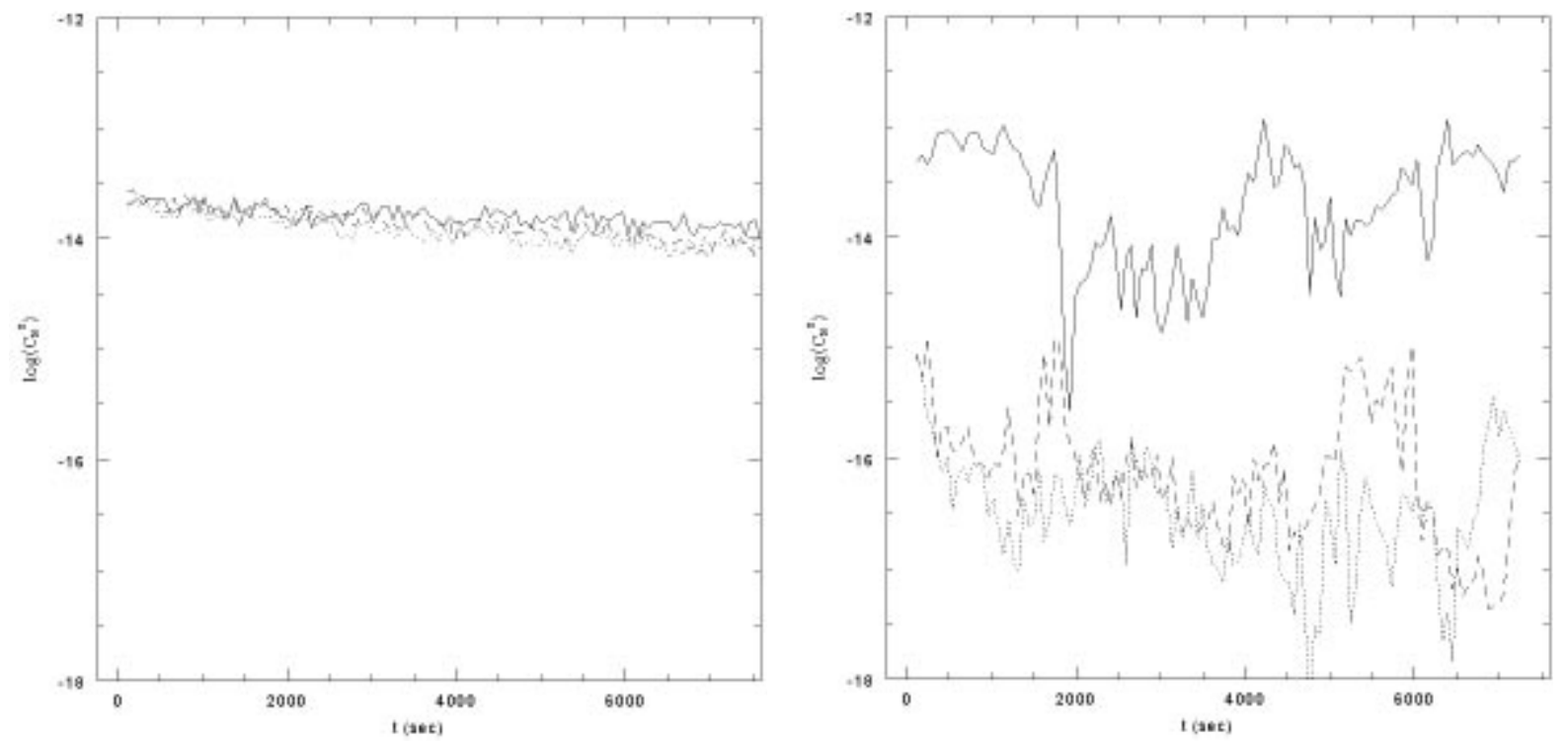

Fig. 2a and b. $C_{N}^{2}$ vs. time: a) for $23 / 05 / 94$, b) for 20/05/94. Solid lines represent the $7 \mathrm{~m}$ level, long dashes the $17 \mathrm{~m}$ level and short dashes the $27 \mathrm{~m}$ level

and La Silla (ESO-VLT working group 1987), where nighttime seeing contributions of $\lesssim 0.1^{\prime \prime}$ were measured over a similar vertical range at the surface. However, the temperature and turbulence structure observed at different times varies markedly, and clear conditions are seen quite often. For example, in Fig. 1b it can be seen that the contribution from the upper layer is $<0.2^{\prime \prime}$ around $35 \%$ of the time.

Two illustrations of the types of conditions are given in Fig. 2.

The first example shows practically identical microthermal variations at each of the three levels, with an average value $\varepsilon=0.53^{\prime \prime}$ over this run. The weather on this day was quite hazy with occasional blowing snow, and a very small temperature inversion measured between $7-$ $27 \mathrm{~m}\left(<0.5^{\circ} \mathrm{C}\right)$. Under these particular conditions we thus have quite even mixing of the atmosphere up to at least $27 \mathrm{~m}$, and possibly much higher, which indicates poor conditions for astronomy.

The second example shows conditions where the microthermal turbulence decreased rapidly from 7 to $27 \mathrm{~m}$. The average seeing for the lower and upper slices was $0.117^{\prime \prime}$ and $0.019^{\prime \prime}$ respectively. The weather conditions were very clear, with a significant temperature inversion (around $4^{\circ} \mathrm{C}$ ) over the height of the mast. The difference between the raw output at each of the levels can be seen quite clearly. It is evident here that the turbulent layer terminates somewhere between 7 and $17 \mathrm{~m}$ and, barring the presence of a second layer above the height of the mast, the surface layer contribution to the seeing above this point is very low.

These examples illustrate the importance of the behaviour of the temperature inversion to surface layer see- ing at the South Pole. Other parameters such as the wind speed and direction, air pressure and the Richardson number (which is derived from temperature and wind speed differentials) do not seem to have any consistent relationship with the turbulence. Analysis of the short timescale fluctuations reveals a strong relationship between the strength and frequency of the vertical temperature changes on a scale of $10 \mathrm{~m}$, and the corresponding turbulence measurements. There appear to be variations in the outer scale of the turbulence, and in its power spectrum corresponding to different conditions. This is observed by comparing the relative intensities of the high frequency temperature fluctuations on the $0.3 \mathrm{~m}$ and $10 \mathrm{~m}$ scale. If the surface layer contribution turns out to be a significant component of the overall seeing, this information will give a very valuable indication of the likely strength and structure of the turbulence in given weather conditions.

Here we will outline some of the major effects observed. The data fit roughly into four categories, as described in Table 2.

The first case in the table represents a large proportion of the runs where poor seeing was measured. At these times the $\varepsilon$ and $\Delta T$ measurements were highly correlated, as were their relative strengths between levels. In fact the seeing values obtained by simply analysing the vertical temperature fluctuations (although these sensors are not as sensitive to high frequency variations) are very close to those derived from measurements on a $0.3 \mathrm{~m}$ scale, indicating that $L_{0}$, the outer scale, extends to at least $10 \mathrm{~m}$.

In the second case, while the temperature inversion and optical turbulence measurements are both very low and stable, the corresponding weather conditions are 
Table 2. The four characteristic types of data observed from the mast. The subscripts 1 and 2 refer to the upper (17-27 m) and lower $(7-17 \mathrm{~m})$ levels respectively. $\bar{\varepsilon}$ is the mean value of the seeing

\begin{tabular}{|c|c|c|c|c|}
\hline & $\varepsilon$ & $\Delta T\left({ }^{\circ} \mathrm{C}\right)$ & Comments & Fraction of Data \\
\hline i) & $\varepsilon \gtrsim \bar{\varepsilon}$ & $1 \lesssim \Delta T_{1,2} \lesssim 2$ & $\begin{array}{l}\text { Average to poor seeing. Can occur in all types of weather. } \\
\varepsilon_{1} / \varepsilon_{2} \sim \Delta T_{1} / \Delta T_{2}\end{array}$ & $40 \%$ \\
\hline ii) & $\varepsilon \ll \bar{\varepsilon}, \varepsilon_{1} \simeq \varepsilon_{2}$ & $\Delta T \lesssim 0.5$ & Very good seeing. Usually occurs during bad weather. & $20 \%$ \\
\hline iii) & $\varepsilon_{1}<\varepsilon_{2}$ & $\Delta T_{1} \ll \Delta T_{2}$ & $\begin{array}{l}\text { Generally good weather, large uneven inversion. Good seeing } \\
\text { above } \sim 17 \mathrm{~m} \text {. }\end{array}$ & $20 \%$ \\
\hline iv) & $\varepsilon_{1}<\varepsilon_{2}$ & $\Delta T_{1}>\Delta T_{2}$ & $\begin{array}{l}\varepsilon_{1} / \varepsilon_{2}<\Delta T_{1} / \Delta T_{2} . \text { Good weather, large, uneven inversion, } \\
\text { temp. gradient increasing with height. Good seeing, esp. above } \\
\sim 17 \mathrm{~m} .\end{array}$ & $10 \%$ \\
\hline
\end{tabular}

almost always poor for astronomy, with significant cloud cover and occasional lower level blowing snow, which evidently suppresses turbulent mixing of the atmosphere in the surface layer.

The third case shows the most common example of the occurrence of low optical turbulence simultaneously with good weather. Here again the behaviour of $\varepsilon$ is closely linked with $\Delta T$.

In the fourth (less common) case we also observe a small seeing contribution in conjunction with good weather. This appears to represent an entirely different surface layer structure, since the gradient of the temperature inversion increases over the height of the mast.

Most of the data fit broadly into one of the cases outlined in Table 2 . The remaining $10 \%$ of data runs generally contained transitions from one state to another over the course of the run, which caused $\varepsilon$ and $\Delta T$ values intermediate between two of these cases.

This information may help us to determine how often good seeing occurs at the South Pole, to the extent that it can be predicted by the temperature gradients in the inversion layer (which are much easier to measure than the microthermal behaviour, and for which historical data are available). We need to know, of course, the contribution from the rest of the boundary layer and the free atmosphere at these times. Acoustic sounding studies (Neff 1981) have been performed at the South Pole which indicate a varying inversion height of $\sim 50-500 \mathrm{~m}$, within which the turbulence structure may be quite complex. These studies also identify a number of classes of characteristic conditions, and their relation to the height and structure of the temperature inversion. More detailed analysis of the microthermal data is in progress with the aim of identifying common meteorological features and making predictions about the strength of the turbulence at higher levels. Data from the 1995 balloon launches will also provide important information about the turbulence structure from $\sim 100 \mathrm{~m}$ to the top of the boundary layer.

\section{Conclusions}

These results summarise the data collected during the 1994 winter season at the South Pole. Although the measured seeing contribution from the atmosphere up to 27 $\mathrm{m}$ is very large on average, the optical turbulence often decreases significantly over the height of the mast. If the results from microthermal balloon launches in 1995 are encouraging with regard to the free atmosphere and upper boundary layer seeing, there may be substantial benefits in placing telescopes on raised platforms.

Our analysis shows that on average around $0.6^{\prime \prime}$ of seeing occurs in the lowest $27 \mathrm{~m}$ of the atmosphere, with much lower values in favourable conditions. However, it remains to be determined how often these conditions occur, as well as the behaviour of the rest of the surface layer at these times.

Acknowledgements. It is a pleasure to acknowledge assistance from Al Harper and Bob Pernic from CARA, and John Storey from the University of New South Wales. We are grateful to Peter Gillingham for his role in the development of this collaboration. Logistical support at the South Pole was provided by the US National Science Foundation. Funding for all instrumentation was provided by l'Institut National des Sciences de l'Univers, France. Support from the Australian Department of Industry Science and Technology's Bilateral Program is gratefully acknowledged.

\section{References}

ESO-VLT working group on site evaluation, 1987, VLT report No. 55.. In: Sarazin M. (ed.)

Ashley M.C.B., Burton M.G., Lloyd J.P., Storey J.W.V., 1995, SPIE 2552, 33 
Ashley M.C.B., Burton M.G., Storey J.W.V., Bally J., Briggs J.W., Harper D.A., Lloyd J.P., 1996, PASP (to be published July)

Burton M.G., Aitken D.K., Allen D.A., et al., 1994, Proc. Astron. Soc. Aust. 11, 127

Dierickx P., 1992, J. Mod. Opt. 39, 569

Fried D.L., 1966, J. Opt. Soc. Am. 56, 1372

Gillingham P.R., 1993, ANARE Res. Notes 88, 290, Australian Institute of Physics 10th Congress, University of Melbourne, February 1992 (publications of the Antarctic Division)

Neff W.D., 1981, An Observational and Numerical Study of the Atmospheric Boundary Layer Overlying the East Antarctic Ice Sheet (PhD Thesis), Wave Propagation Laboratory, Boulder Colorado U.S.A.

Obukhov A.M., 1949, Izv. Akad. Nauk SSSR, Ser Geograf. Geofis. 13, 58
Roddier F., 1981, Prog. Opt. 19, 281

Schwerdtfeger W., 1984, Weather and Climate of the Antarctic. Elsevier Science Pub. Co. NY

Storey J.W.V., Ashley M.C.B., Burton M.G., 1996, Publ. Astron. Soc. Aust. 13, 35

Tatarski V.I., 1961, Wave Propagation in a Turbulent Medium. McGraw-Hill, New York

Vernin J., 1994, Recherche de site pour l'astronomie en Antarctique, Colloque Acad. Sci. Paris, 16-18 Dec. 1992, 92-96

Vernin J., Marks R., Ashley M.C.B., Azouit M., Briggs J.W., Burton M.G., Manigault J.F., 1994, Optical Turbulence at the South Pole: First Measurements and Future Plans, XXIIIrd SCAR Meeting, Rome, Aug. 29-Sep. 1

Vernin J., Muñon-Tuñóz C., 1992, A\&A 257, 811

Vernin J., Muñon-Tuñóz C., 1994, A\&A 284, 311 